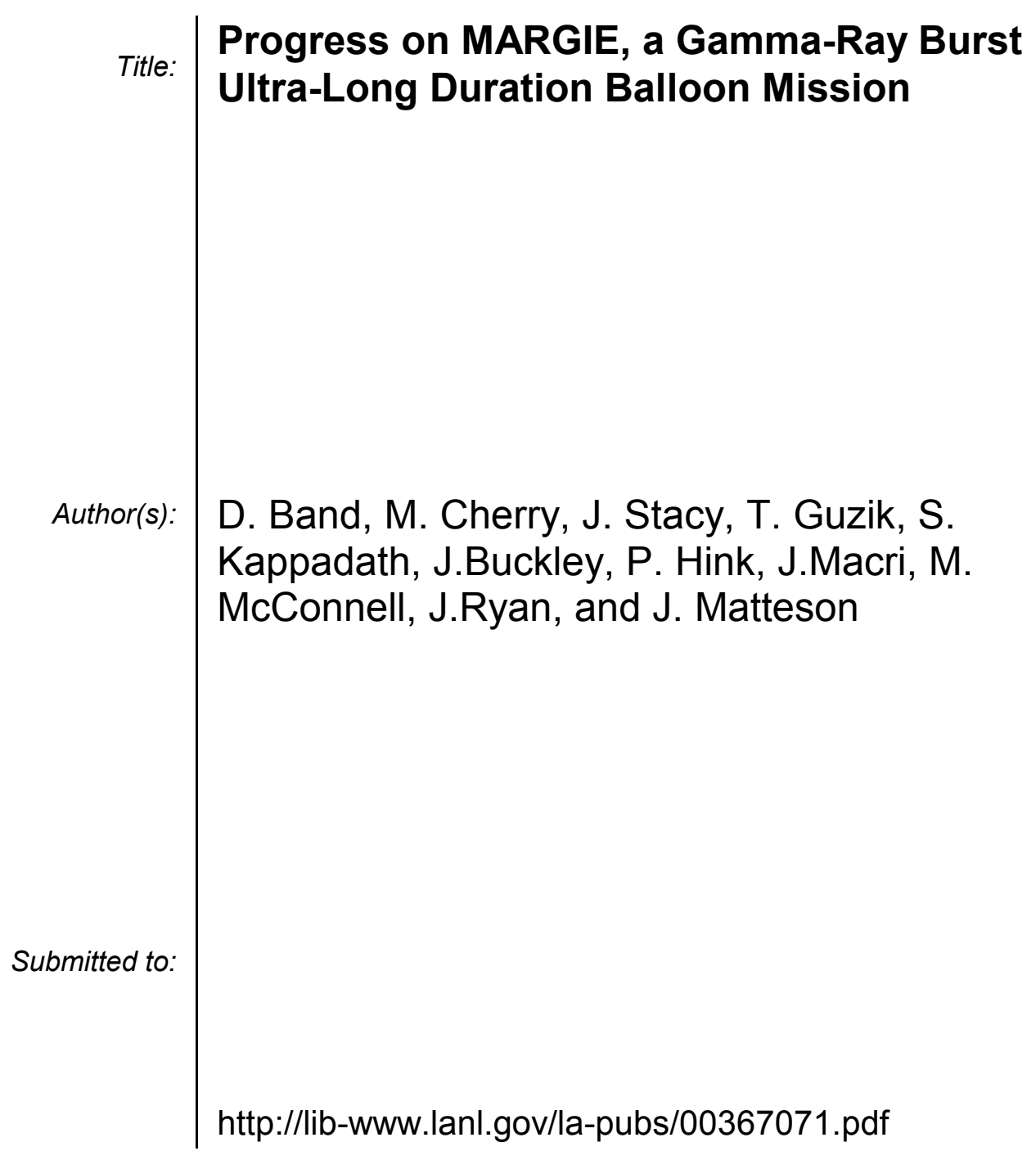

Los Alamos National Laboratory, an affirmative action/equal opportunity employer, is operated by the University of California for the U.S. Department of Energy under contract W-7405-ENG-36. By acceptance of this article, the publisher recognizes that the U.S. Government retains a nonexclusive, royaltyfree license to publish or reproduce the published form of this contribution, or to allow others to do so, for U.S. Government purposes. Los Alamos National Laboratory requests that the publisher identify this article as work performed under the auspices of the U.S. Department of Energy. Los Alamos National Laboratory strongly supports academic freedom and a researcher's right to publish; as an institution, however, the Laboratory does not endorse the viewpoint of a publication or guarantee its technical correctness. 


\title{
Progress on MARGIE, a Gamma-Ray Burst Ultra-Long Duration Balloon Mission
}

\author{
D. Band ${ }^{1}$, M. Cherry ${ }^{2}$, J. Stacy ${ }^{2}$, T. Guzik ${ }^{2,3}$, S. Kappadath ${ }^{2}$, J. Buckley ${ }^{4}$, \\ P. Hink ${ }^{4}$, J. Macri ${ }^{5}$, M. McConnell ${ }^{5}$, J. Ryan ${ }^{5}$, and J. Matteson ${ }^{6}$ \\ 1 X-2, Los Alamos National Laboratory, Los Alamos, NM, 87545, USA \\ 2 Louisiana State University, Baton Rouge, LA, 70803, USA \\ 3 Southern University, Baton Rouge, LA, 70813, USA \\ 4 Washington University, St. Louis, MO, 63130, USA \\ 5 University of New Hampshire, Durham, NH, 03824, USA \\ 6 CASS, University of California at San Diego, La Jolla, CA, 92093, USA
}

\begin{abstract}
We are designing the Minute of Arc Resolution Gamma-ray Imaging Experiment (MARGIE) as a 100 day Ultra Long Duration Balloon (ULDB) mission to: a) detect and localize gamma-ray bursts; and b) survey the hard X-ray sky. Major advances in designing the CZT detectors increase the senstitivity to higher energy. Design of the gondola has also progressed.
\end{abstract}

\section{Introduction}

The MARGIE project is a synthesis of advanced mask construction, detector material and gondola control technologies under development at the various institutions in this collaboration. In addition to performing astrophysically relevant observations, MARGIE will be a test bed for these new technologies. Since the burst-detection capabilities were reported at a previous gamma-ray burst meeting[1], here we provide a progress report on technical advances.

The MARGIE mission will consist of a central imager with a small $\left(8.3^{\prime}\right.$ half angle) field-of-view (FOV) and 4 large (26.1' half angle) FOV detectors whose orientations will be offset from that of the central detector. With areas of $\sim 1900 \mathrm{~cm}^{2}$, the detector plane will most likely be Cadmium-Zinc-Telluride (CZT), with segmented CsI scintillators as a backup. With $0.83 \mathrm{~mm}(0.5 \mathrm{~mm})$ pixels for the central (side) detector and a detector-mask distance of $150 \mathrm{~cm}$ $(45 \mathrm{~cm})$, the geometric resolution will be $1.9^{\prime}\left(3.8^{\prime}\right)$. Imaging will be achieved using a tungsten coded mask consisting of a 2 dimensional URA pattern with 2 cycles in each direction. The burst position will be calculated onboard and disseminated in near-real time, while information about every count will be stored for further analysis.

\section{The Detector Plane}

We plan to use the CZT detector design developed at the Universities of New Hampshire and Montreal[2,3]. In this design, the cathode and anode are on 
the source and back sides of the detector, respectively. The anode consists of orthogonal coplanar electrodes. Each of the $\mathrm{N}$ rows of anode electrodes consists of $\mathrm{N}$ interconnected pixels, while each of the $\mathrm{N}$ columns is a single conducting strip. The pixels are circles punched out of the conducting strips which make up the columns, with a contact bonded to the surface of the CZT in the middle of the pixel. This geometry permits an $\mathrm{N} \times \mathrm{N}$ pixel array to be read out by $2 \mathrm{~N}$ electronic channels, reducing the complexity of the electronics. A gamma ray absorbed in the CZT produces electron-hole pairs. The anode geometry relies on the relatively mobile electrons for the energy and row/column location measurements. Processing of the shape of the strip (column) signal exploits hole trapping in the CZT to measure the depth of interaction. Since the strips surrounding the pixels are biased at a voltage intermediate between the cathode on the source side and the pixels, the pixels collect the electrons but the strips surrounding the pixels register a transient signal as the electron cloud approaches the anode plane. Since the electrons produce a signal in more than one strip or row of pixels, the position of the absorbed photon can be resolved to $0.1 \mathrm{~mm}$ along a row and $0.3 \mathrm{~mm}$ along a strip, even though the pixels are $1 \mathrm{~mm}$ across. The depth can be resolved to less than $1 \mathrm{~mm}$. Because the horizontal position is determined by the electrons at the anode, and detection of the holes is secondary, the CZT can be thicker (up to $10 \mathrm{~mm}$ ), increasing the detector's high energy efficiency (e.g., to $E>1 \mathrm{MeV}$ ). The gamma-ray energy deposited is measured by the strength of the electron signal. The measured spectral resolution is currently $5.7 \%$ at $60 \mathrm{keV}, 2.6 \%$ at $122 \mathrm{keV}$ and $0.94 \%$ at $662 \mathrm{keV}$, within a factor of 3 of the theoretical limit.

The technology of packaging the CZT detectors and bonding the leads connecting the pixels has been developed and demonstrated. The major impediment is the cost of the CZT $\left(\$ 3000 \mathrm{~cm}^{-2}\right)$; this cost will undoubtedly come down with time, particularly for a large quantity. However, as an alternative we have developed and will test segmented BGO scintillator detectors.

\section{The Gondola}

A ULDB is essentially a spacecraft mission without the paperwork. We will use the experience of the team members in other ULDB projects, specifically the TIGER program at Washington University which will be the first ULDB flight. The MARGIE detectors will be oriented vertically within a spherical KEVLAR pressure shell. Such a pressure shell was successfully flown on the recent 17day long duration Antarctic flight of the ATIC cosmic ray experiment[3]. This pressure shell will sit within a frame of I beams. Thus the central detector will be constrained to point vertically. The rotor connecting the gondola to the balloon will provide the azimuthal orientation. Sub-arcminute resolution aspect determination will result from fiber optic gyroscopes, CCD cameras and a GPS receiver. The data will be stored primarily on board with the ability to upload commands and download data when the mission is within line-of-sight of a groundstation or a chase aircraft. However, MARGIE will also have a TDRSS 
link which will be used for time-critical communication, such as downloading burst positions (which can then disseminated through GCN).

The science package (detectors, pressure vessel, frame but not NSBF components) will weigh $\sim 950 \mathrm{~kg}$. The weight and power, as well as the payload durability, all meet ULDB requirements.

\section{Burst Sensitivity}

We discussed MARGIE's burst sensitivity previously [1], and thus here we only present a summary. Bursts will be detected by a statistically significant increase (e.g., by $5 \sigma$ ) in the count rate. We find a BATSE-equivalent (i.e., 50$300 \mathrm{keV})$ threshold peak flux of $\psi_{\text {cen }}=0.209 t^{-1 / 2}\left(\sigma_{b} / 5\right) \mathrm{ph} \mathrm{s}^{-1} \mathrm{~cm}^{-2}$ and $\psi_{\text {side }}=$ $0.376 t^{-1 / 2}\left(\sigma_{b} / 5\right) \mathrm{ph} \mathrm{s}^{-1} \mathrm{~cm}^{-2}$ for the central and each side detector, respectively. Based on the burst rate, the FOV, and a mission duration of $M \sim 100$ days, we find that MARGIE should detect $N_{\text {cen }}\left(>\psi_{\text {cen }}\right)=1.1 t^{0.4}\left(\sigma_{b} / 5\right)^{-0.8}(M / 100 \mathrm{~d})$ and $N_{\text {side }}\left(>\psi_{\text {side }}\right)=7.1 t^{0.4}\left(\sigma_{b} / 5\right)^{-0.8}(M / 100 \mathrm{~d})$ bursts in the central and each of the side detectors, respectively. Accounting for overlapping FOVs, $\sim 22$ bursts should occur in some detector's fully coded FOV. Of course, the detectors will also detect bursts in the partially coded FOVs. Therefore, we should detect at least 40 bursts. We assume that each detector triggers independently.

For the fully-coded FOV, the brightest burst expected in 100 days (i.e., $\psi_{b}$ such that $N\left(>\psi_{b}\right)=1$ ) will have a peak flux of $\psi_{b \text {,cen }}=0.25 \mathrm{ph} \mathrm{cm}^{-2} \mathrm{~s}^{-1}$ and $\psi_{b \text {,side }}=4.36 \mathrm{ph} \mathrm{cm}^{-2} \mathrm{~s}^{-1}$. Of course, with the much larger area covered by all 4 side detectors, including the partially coded regions, the "burst of the mission" will probably be somewhat larger.

With the small number of counts accumulated during a burst and distributed over a much larger number of pixels, backprojection will be the most efficient image reconstruction technique to localize the bursts. At the burst threshold there is a $\sim 10 \%$ chance of a spurious localization in a side detector but this probability rapidly becomes negligible for brighter bursts. Similarly, persistent sources in the FOV should not be bright enough to compromise the localizaton, although images before and after the burst should identify such background sources. Sources can be localized to less than the geometric resolution; we anticipate localizations of $\sim 1.7^{\prime}$ in the side detectors.

\section{References}

1. D. Band, et al.: 'MARGIE, a Gamma-Ray Burst Ultra-Long Duration Balloon Mission'. In: Gamma-Ray Bursts, Proc. of the 5th Huntsville Symposium, ed. by R. M. Kippen, R. Mallozzi, and G. Fishman (AIP, New York, 2000), pp. 696-697

2. McConnell, M. L., et al.: 'Three dimensional imaging and detection efficiency performance of orthogonal coplanar CZT strip detectors'. In: Proc. SPIE 4141, 157 (2000)

3. P. Altice, et al.: 'MARGIE'. In: Proc. 33rd COSPAR Scientific Assembly, Warsaw, Poland, 16-23 July 2000 (2001), in press

4. G. Guzik, et al.: Advances in Space Research 19, 711 (1997) 